\begin{tabular}{|c|c|c|}
\hline \multicolumn{2}{|c|}{ Valletta und Floriana (Zentrum) } & $2+076$ Personen \\
\hline $\begin{array}{l}\text { Cospicua } \\
\text { Senglea } \\
\text { Vittoriosa }\end{array}$ & Östliche Vororte & 19 o5o Personen \\
\hline $\begin{array}{l}\text { Hamrun } \\
\text { St. Venera } \\
\text { Marsa } \\
\text { Pietà }\end{array}$ & Westliche Vororte & 3729 o Personen \\
\hline $\begin{array}{l}\text { Gzira } \\
\text { Msida } \\
\text { St. Julians } \\
\text { Sliema }\end{array}$ & Nördliche Vororte & $4951+$ Personen \\
\hline $\begin{array}{l}\text { Paola } \\
\text { Tarxien } \\
\text { Zabar } \\
\text { Kalkara } \\
\text { Luqa }\end{array}$ & Südliche Vororte & to oo1 Personen \\
\hline
\end{tabular}

Die Agglomeration Valletta und Umgebung umfaßt demnach 169931 Einwohner. Für die Stadt Bern wird im Jahre 1965 vergleichsweise eine Zahl von 171000 Einwohnern verzeichnet. Auch anlagemäßig besteht eine gewisse Übereinstimmung zwischen Valletta und Bern: beide liegen auf einer langgestreckten $\mathrm{Halbinsel}$ und sind von Wasser umgeben.

\title{
VALLETTA
}

La capitale de Malte, Valletta, a été fondée en 1566 sur la presqu'île centrale de l'île de Malte. Port important, elle remplit en même temps des fonctions administratives et d'importantes fonctions commerciales. Donc plus de 50\% de la population maltaise - en 1962, Valletta et environs comptaient 169931 habitants - y sont concentrés. Malgré ce phénomène, la ville présente, du fait d'un prestigieux passé, de nombreux palais, églises et d'anciennes demeures seigneuriales, qui lui donnent le caractère d'un centre culturel.

\section{MORPHOLOGISCHE BEOBACHTUNGEN IN OST-NEPAL}

\author{
HANS BOESCH
}

Die folgenden Beobachtungen wurden im Frühjahr 1964 gemacht. Vom 3. bis 19. März hielt ich mich in Nepal auf, um in Zusammenarbeit mit SATA (Swiss Association for Technical Assistance) die vom Geographischen Institut der Universität Zürich eingerichteten meteorologischen Stationen zu besichtigen. $\mathrm{Zu}$ diesem Zwecke flog ich nach kurzem Aufenthalt in Katmandu schon am 5. März in 35 Minuten in dem vom Teamleiter von SATA, Herrn Jenny, pilotierten Champion-Flugzeug zu dem ziemlich genau 100 Kilometer in East No. 2 gelegenen Jiri. Dort befindet sich die damals von Herrn Monsch geleitete Büffelfarm sowie das Spital, welches vom SHAG (Schweizerisches Hilfswerk für Außereuropäische Gebiete) eingerichtet worden ist. Der Rückmarsch nach Katmandu erfolgte vom 11. bis 15. März: in fünf Tagen statt der $35 \mathrm{Mi}$ nuten Flug! Auf diesem Rückmarsch konnte ich einige morphologische Beobachtungen machen, welche ich hier, trotz ihres zufälligen und fragmentarischen Charakters, mitteilen möchte, weil erst wenig bekannt ist. Da der Weg von Katmandu nach Jiri und Those häufig von Wissenschaftern begangen wird - ist es doch der Hauptzugangsweg 
zum Gebiet des Mount Everest -, wird vielleicht der eine oder andere unter ihnen, durch die nachstehenden Bemerkungen angeregt, in Zukunft eingehendere Untersuchungen anstelllen und damit unsere Kenntnisse erweitern.

Über den Hauptzweck meiner Reise liegt ein Bericht vor ${ }^{1}$. Zu Fragen der Morphologie gibt es erst wenige Publikationen, wobei für die hier aufgeworfenen Fragen in erster Linie T. Hagen ${ }^{2},{ }^{3}, \mathrm{H}$. Heuberger ${ }^{4}$ und A. Lombard ${ }^{5}$ zu erwähnen sind.

Hagen gibt einen allgemeinen Überblick über die geologisch-strukturellen Verhältnisse. Meine Reiseroute führte in ost-westlicher Richtung durch die sogenannten Midlands, eine der vier Hauptzonen von Nepal (von S nach N : Terai, Mahabharat-Kette, Midlands, Himalaya-Hauptkette). Wie das Profil zeigt, sind die Höhenunterschiede in den Midlands immer noch sehr groß; es fehlen ihnen aber die Hochgipfel der Hauptketten, wie auch die Zerschluchtung beim Durchbruch der Flüsse durch die Mahabharat-Kette. Der Ausdruck 'midlands' ist deshalb nur im Vergleich mit den anderen Zonen zuläßig; absolut gesehen, ist die Reliefenergie bedeutend. Nach Hagen befinden wir uns längs der Reiseroute im Bereiche zweier flach übereinanderliegender Deckeneinheiten, welche zudem als flache Kuppel emporgestaucht worden waren. Sie zeichnen mit ihren Strukturen den Lauf des Sun-Kosi vor. Hagen nennt diese Struktur die KosiAntiklinale. Die untere Deckeneinheit ist jene der Nawakot-Decken, die höhere, die nördlichen Berggipfel bildend und südlich am Sun-Kosi in die Tiefe eintauchend, die Folge der Katmandu-Decken. Die Gesteine sind durchwegs Ortho- und Para-Gneise.

Heuberger beging die Route 1954 als Mitglied der österreichischen Tscho-OyuExpedition. Seine sorgfältigen kulturgeographischen Beobachtungen längs der Route machen den Hauptteil seiner Arbeit aus, doch finden wir dazwischen eingestreut Bemerkungen und Beobachtungen zur Morphologie von Bedeutung. Um eine gewisse Einheitlichkeit zu erzielen, benutzte ich für die Ortsnamen meistens die Schreibweise von Heuberger. Auskunft über Ortsnamen erhält man durch Befragung der Träger, was zu recht verschiedenen Resultaten führen kann! Nur wenige Ortsnamen wie Dolalghat, Charikot oder Those finden wir in den erhältlichen Karten.

Von allen vorliegenden Berichten ist derjenige von Lombard mit Bezug auf geologisch-morphologische Fragen am besten dokumentiert. Obwohl das Hauptgewicht auf der Untersuchung des Khumbu-Gebietes liegt, enthalten die ersten zwölf Seiten für die Route Katmandu-Those zahlreiche morphologisch bedeutsame Angaben mit präzisen Profilen und Beschreibungen.

Der von mir gewählte Weg ist, wie erwähnt, der Hauptzugangsweg zum Mount Everest und in den Osten von Nepal. Zwischen Katmandu und Banepa konnte er, wenn auch über teilweise unglaublich schlechte Straßen, mit dem Jeep befahren werden. Die mit technischer und finanzieller Hilfe der Chinesen zu bauende Straßenverbindung von Katmandu nach Lhasa war erst in Angriff genommen worden. Bis Paksal konnten schon leichte Wagen verkehren; bis Dolalghat war der Aushub ausgeführt, doch fehlten noch die Kunstbauten. Dies bedeutete, daß von Jiri bis Banepa der Trägerpfad benutzt werden mußte. Verlauf und Höhenverhältnisse zeigt Fig. 1. Ein großer Teil der nepalischen Bevölkerung ist ständig unterwegs, meist um im Auftrage die Gütertransporte zu besorgen. Traglasten von $30-40 \mathrm{~kg}$ sind normal, oft sind sie aber auch bedeutend größer.

1 Boesch Hans: Zwei Jahre Wetterbeobachtungen in Nepal (1961-1963). Geogr. Helv., 1964, 170-178, 2 Fig., 3 Tab.

2 Hagen Toni: Das Gebirge Nepals. Alpen, 1956. (Vier Fortsetzungen)

3 Hagen Toni: Über den geologischen Bau des Nepal-Himalaya. Ber. St. Gall. Naturw. Ges., Bd. 76, 1959. 120 S., 10 Fig. 9 Taf.

4 Heuberger Helmut: Der Weg zum Tscho Oyu. Mitt. Geogr. Ges. Wien, Bd. 98, 1956, 28 S., 6 Fig., 1 Taf., 12 Bilder

5 Lombard A.: Un itinéraire géologique dans l'Est du Nepal (massif du Mount Everest) Denkschrift SNG, Bd. 77, Abh. 1, 1958. 107 S., 126 Fig. 


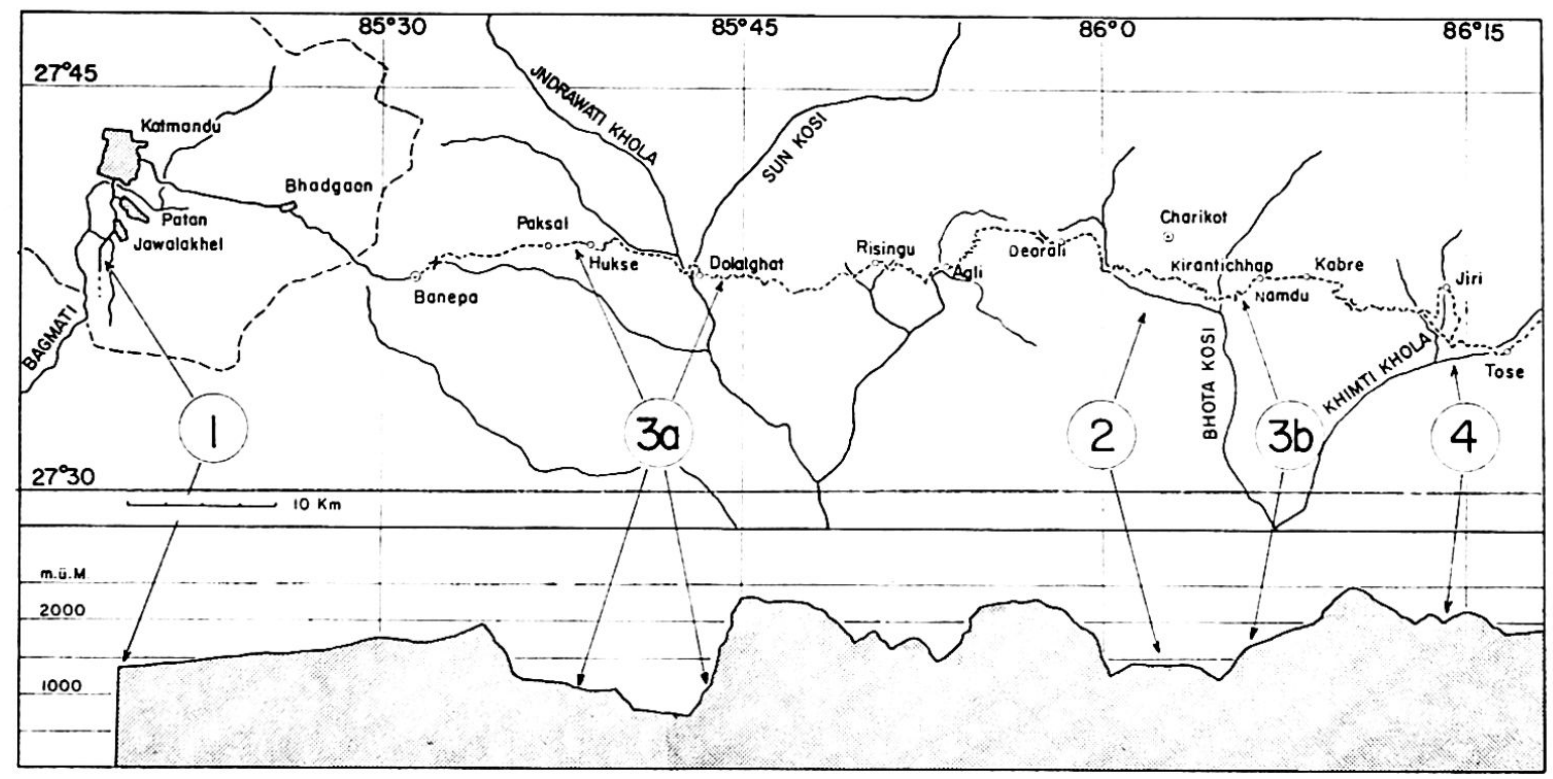

Figur 1 Routenskizze und Profil Katmandu-Jiri und Those. Die Zahlen 1, 2, 3a, 3 b, 4 beziehen sich auf den Text.

Allgemein verwendet werden Tragkörbe, welche mit Schulterriemen und Stirnband gehalten werden. Außerdem benützen die Träger einen krückenartigen Stock, auf den sie bei kurzen Halten den Tragkorb aufstützen. Bei längeren Halten stellen sie die Last an einem der zahlreich vorhandenen, bankartigen Mäuerchen am Wegrande ab. Ich nahm zur Begleitung einen einzigen Träger mit. Aufbruch war jeweilen beim Morgengrauen, kurz nach 6 Uhr. Mittags wurde ein Halt von etwa 2 Stunden eingeschaltet. Abends wurde irgendwo, wo Holz und Wasser vorhanden waren, Rast gemacht und im Freien kampiert. Der klare Nachthimmel mit einem ihn regelmäßig durchlaufenden Satelliten bleibt unvergeßlich. Proviant mußte mitgenommen werden; unterwegs waren kaum Lebensmittel zu finden, weil zu jener Zeit Hunger herrschte. Dagegen kam man jeden Tag an einem oder mehreren Verkaufsläden vorbei, wo Streichhölzer, Zigaretten usw. zu kaufen waren.

Am 11. März konnte ich, begleitet von meinem Träger, Jiri um 07.45 verlassen. Schon um 08.00 erreichten wir den flachen Höhenrücken zwischen dem Jiri- und SikriTal, wo einmal in der Woche der große Markt der Region im freien Felde stattfindet. Von hier hat man einen ausgezeichneten Rückblick auf das morphologisch interessante Tal von Jiri (Fig. 1; Beispiel 4). Da der Hauptpfad nicht über Jiri führt (siehe Kartenskizze Fig. 1), ist es in der oben erwähnten Literatur unberücksichtigt geblieben. Dank der Tätigkeit des SHAG besteht heute eine gute photographische und kartographische Dokumentation über das Jiri-Tal.

Während in allen anderen Tälern, welche die Route quert, die junge Erosion rückwärtsgreifend tief einschnitt und unwegsame Schluchten schuf, finden wir im Tal von Jiri einen weiten Talboden. Von den Bergen im Talhintergrund zieht ein breiter, heute völlig überwachsener großblockiger Schuttstrom, welcher das Tal füllt, herunter. Diese Schuttfüllung schafft ein kastenförmiges Querprofil des Tales mit flachem bis leicht gewölbtem, talauswärts geneigtem Talboden. Die rückwärtsgreifende Erosion hat hier diese Schuttmassen noch nicht weggeräumt; das obere Ende der Erosionsschlucht erreicht nur gerade noch den untersten Teil des Jiri-Tales. Dazu kommt im unteren Abschnitt des weiten Tales eine Schüttung von Gehängematerial (vermutlich eine Rutschung des rechten Talhanges), welche den Talbach aufstaut und die Bildung eines weiten, fast flachen und ursprünglich stark versumpften Aufschüttungsboden verursacht. Aus diesem Grunde besteht hier auch die Möglichkeit zur Anlage eines kleinen Flug- 


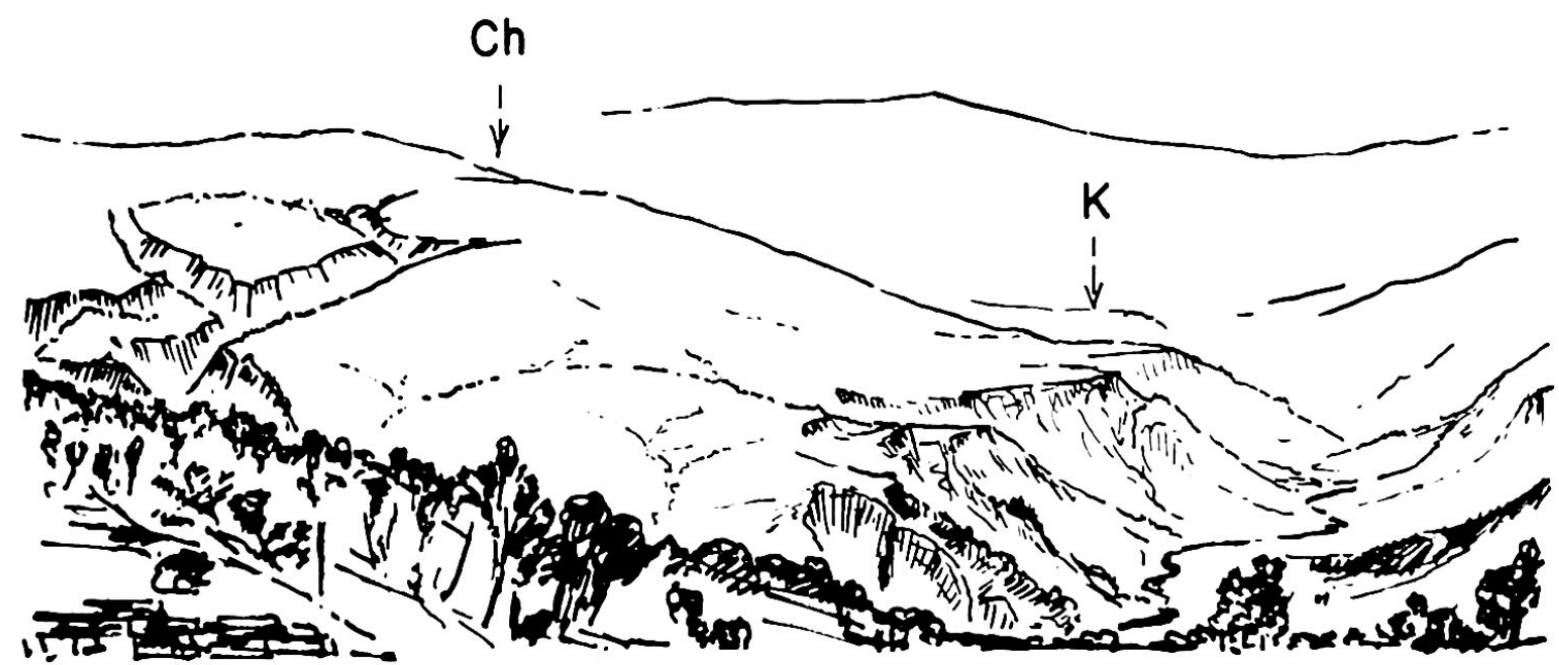

Figur 2 Rückblick vom Aufstieg gegen Deorali in östlicher Richtung $(\mathrm{Ch}=$ Charıkot, $\mathrm{K}=$ Kirantichhap). Hinter der letzten Kulisse liegt das Tal von Sikri und Jiri.

platzes. Außerdem sind diese morphologischen Verhältnisse für die ausgedehnten Weide- und Futterflächen der Büffelfarm von Jiri verantwortlich. Die morphologische Situation von Jiri ist somit eine ganz besondere und einzigartige.

Unsere Deutung geht dahin, daß es sich um eine spätpleistozäne Blockschuttstreuung handelt, die heute inaktiv geworden ist. In andern Tälern dürfte die Situation ursprünglich eine ähnliche gewesen sein, wobei aber die nachpleistozäne Erosion zu einer völligen Ausräumung geführt hat.

Schon das benachbarte Sikri-Tal, wo wir die Hauptroute erreichten, hat einen anderen morphologischen Charakter mit schluchtartigem unterstem Talabschnitt. Nun kommt von 09.30 bis 11.00 der erste steile Aufstieg zum Kaileswar-Paß - wie viele solche Anstiege gibt es noch in den folgenden Tagen! - gefolgt von einem ebenso steilen Abstieg auf die zwischen 2000 und ca. $1800 \mathrm{~m}$ ü. M. gelegene prächtige Systemsterrasse von Yaksa, Kabre und Namdu. Um 16.00 machten wir am Ausgange von Namdu halt für die erste Nachtrast.

Um 06.30 brachen wir wieder auf und erreichten um 07.30 den Bhota Kosi, über welchen eine ausgezeichnete, hochgespannte Hängebrücke (erbaut von der Firma Henderson \& Co., Aberdeen) führt. Etwa $200 \mathrm{~m}$ über dem Talgrund versteilte sich beim Abstieg das Gehänge unvermittelt: die Systemsterrasse von Kabre ging hier plötzlich in die jüngste Schluchtphase über. Kurz vorher waren beidseits des Weges Roterdeböden zu beobachten (Fig. 1; Beispiel 3b), welche später (Beispiel 3a) besprochen werden sollen. Schon um 09.00 erreichten wir Kirantichhap, wo zum einzigen Male auf der ganzen Reise ein Polizist sich nach unseren Absichten erkundigt; außerdem konnten wir zwei Eier kaufen! Im späteren Vormittag führte der Weg durch stark rutschendes Gelände horizontal dahin. Primär sind diese Rutschungen (Fig. 1; Beispiel 2) zweifellos durch das Talgewässer verursacht. Die Höhenlage gestattet den Anbau von Reis. In der jetzigen Jahreszeit lagen die Felder mit Ausnahme der Saatfelder noch brach. In die späteren Saatfelder wurde aber schon Wasser durch kleine Kanäle geleitet, wodurch sekundär die Gehängerutschungen gefördert wurden. Mittagshalt machten wir am rechten Nebenfluß des Bhota Kosi in prächtiger Waldschlucht. Am frühen Nachmittag war die Schlucht überwunden und das schon besprochene, höher gelegene Niveau wieder erreicht, welches jenem von Kabre entspricht. Von hier aus ist Fig. 2, welche auf Grundlage von Photographien entworfen wurde, gezeichnet. Sie soll die Bedeutung der jüngsten Schluchtphase veranschaulichen, welche äußerst aktiv in die höheren Flachformen einschneidet und in einem in den Alpen nicht bekannten Ausmaße zur ständigen Verrutschung der Talhänge führt. Es handelt sich dabei um eine allgemeine Erscheinung, 


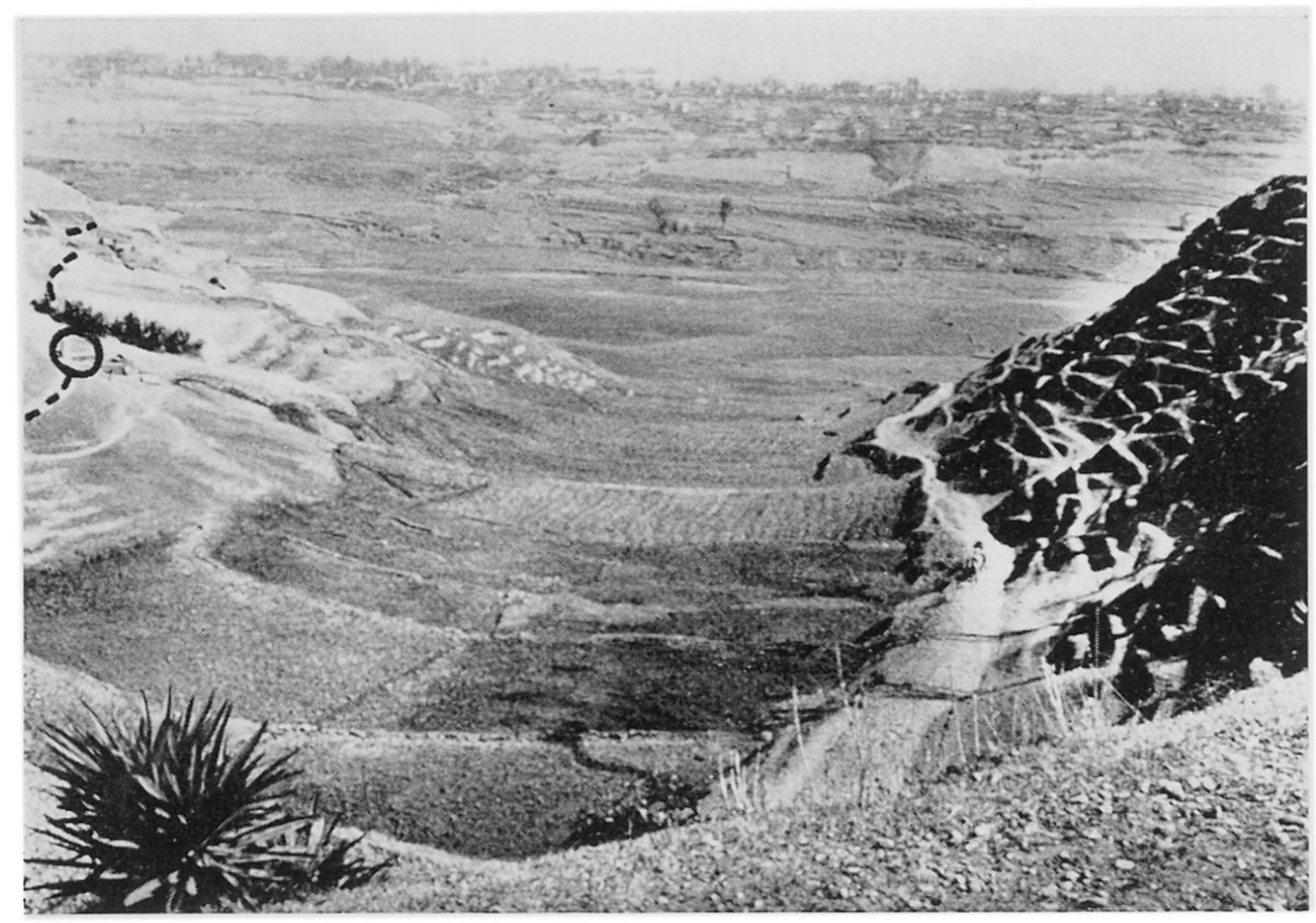

Figur 3 Kleines Seitental des Nakhu Khola, südlich Jawalakhel-Katmandu. Siehe Beschreibung im Text. Aufnahme: HB 4.3.64

die aber hier besonders eindrücklich beobachtet werden kann. Um 16.00 wurde unterhalb des Passes von Manga-Deorali angehalten.

Der folgende Tag führte über den $\mathrm{Pa} \beta$ von Manga-Deorali in das wild durchtalte obere Einzugsgebiet eines Zuflusses des Sun-Kosi ; das Nachtlager wurde kurz nach Risingu bei Uapal in nächster Nähe des Flusses, gegenüber einer bis tief in die Nacht arbeitenden Getreidemühle, aufgeschlagen.

Am vierten Tag, von Uapal bis Dolalghat, waren die morphologischen Verhältnisse grundverschieden. Nach einem etwa einstündigen steilen Aufstieg wurde ein langgestreckter Höhenrücken erreicht, über welchen in leichtem Auf und $\mathrm{Ab}$ der Pfad in etwa $2200 \mathrm{~m}$ Höhe dahinführte. Stundenlang findet sich hier kein Wasser, bis der lange Abstieg nach Dolalghat einsetzt. Wir erreichten diesen Ort schon um 15.00. In Dolalghat war es möglich, Einkäufe (Reis, Zucker, Tee) zu tätigen; der Ort besteht hauptsächlich aus Ladengeschäften.

Morphologisch sind die im Abstieg gegen Dolalghat (Fig. 1; Beispiel 3a) auftretenden Terrassen, zusammen mit ihren alluvialen Bedeckungen, von größtem Interesse. Sowohl Heuberger wie vor allem auch Lombard haben sie beschrieben. Die Höhenangaben weichen etwas voneinander ab. Ich selber hatte keinen brauchbaren Höhenmesser. Ich stützte mich deshalb auf die Angaben von Lombard, der die Terrassen in Höhen von $1100 \mathrm{~m}, 1030$ und $840 \mathrm{~m}$ über dem bei $580 \mathrm{~m}$ ü. M. liegenden Flußniveau feststellte. Ich kann die Angaben von Lombard nur bestätigen (p. 7 ) : «Chacune de ces terrasses correspond à un niveau d'accumulation. Son matériel est tantôt de l'alluvion tantôt du sol d'altération, souvent coloré de rouge, ce que je rapporte à la latérisation, sans impliquer par là que le processus ait atteint un stade avancé.» Auf p. 6 beschreibt er die Terrasse von $840 \mathrm{~m}$ im Detail und fügt bei: «A la latérite se mèlent de gros boulets pro- 


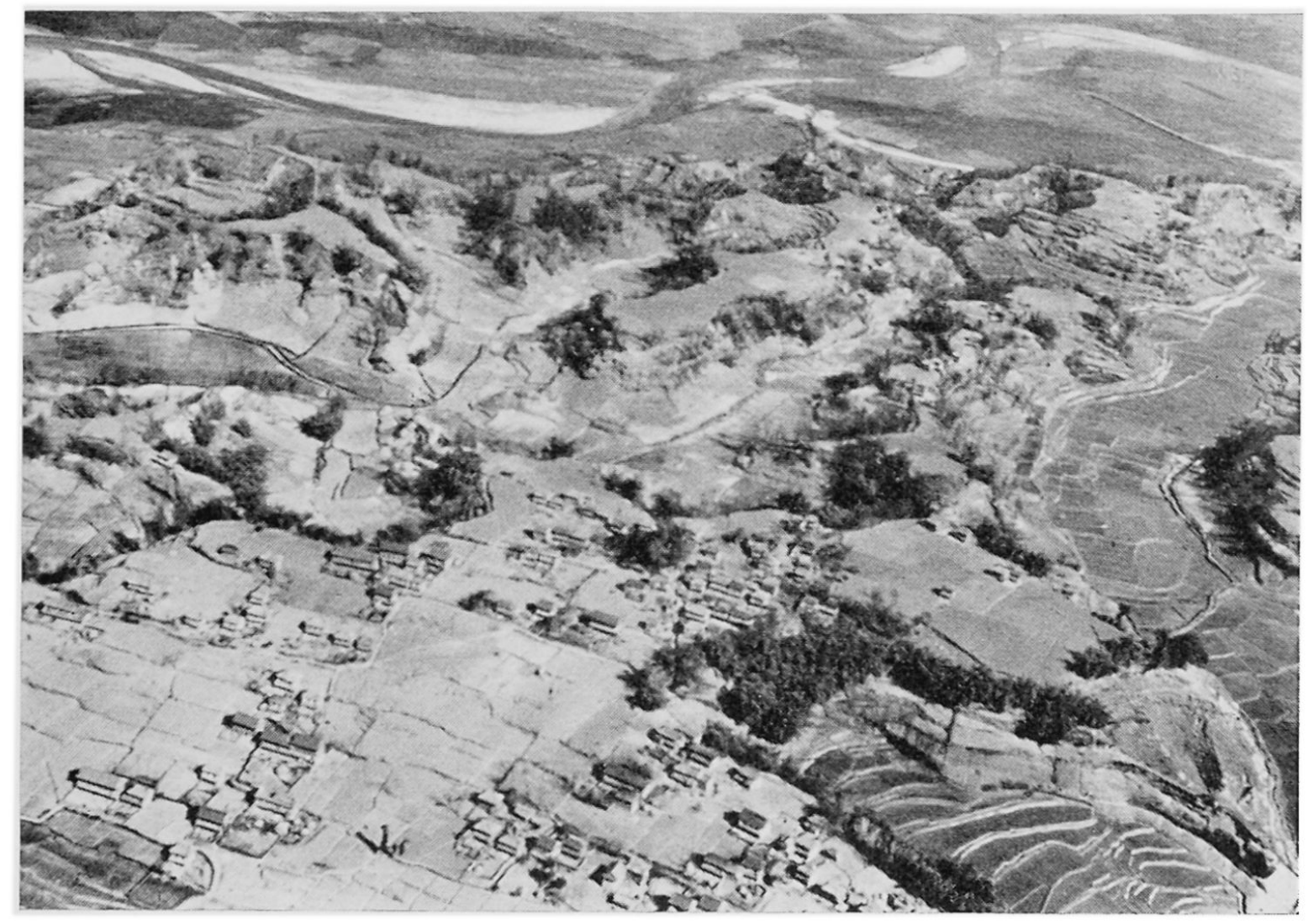

Figur 4 Flugaufnahme eines Dorfes im Valley, östlich von Katmandu. Die geologisch-morphologische Situation ist dieselbe wie in Figur 3; die Landnutzung ist im Text beschrieben. Aufnahme: HB 5.3.64

bablement alluviaux et anciens». Ich möchte beifügen, daß im allgemeinen diese Gerölle, welche einige Dezimeter im Durchmesser erreichen können, eine tief schwarze Verwitterungsrinde besitzen und mich in jeder Beziehung an Produkte arider Verwitterung erinnerten. Leider war eine Probenmitnahme nicht möglich, doch scheint mir hier der Ansatzpunkt für klimamorphologische Untersuchungen in Verbindung mit der Talgenese zu liegen.

Ich erwähnte solche Roterdebildungen schon im Abstieg zum Bhota Kosi unterhalb Namdu. Wir sollten sie erneut in ähnlicher Höhenlage am letzten Tag finden. Dolalghat wurde schon um 05.30 verlassen. Zuerst dem Trassee der sogenannten 'ChinesenStraße' folgend, dann steil ansteigend, erreichten wir bei Hukse in höherem Niveau erneut ausgedehnte Vorkommen von Roterdeböden. Leider drängte die Zeit derart, daß keine eingehendere Untersuchung möglich war. Erst später fand ich bei Lombard eine Angabe, daß die die terra rossa tragenden Terrassen jungtektonisch verstellt seien. Wir mußten um 15.00 Banepa erreichen, um die letzte Fahrtmöglichkeit nach Katmandu nicht zu verpassen. Kurz nach 17.00 trafen wir in Katmandu ein und erreichten gegen 18.00 im Vororte Jawlakhel Ekanta Kuna, das Haus der SATA.

Die Figuren 3 und 4 illustrieren die Situation im Becken von Katmandu, dem sogenannten 'Valley'. Es handelt sich um ein intramontanes, tektonisch geschaffenes Becken, welches zuerst von einem See erfüllt war und heute durch den Bagmati entwässert wird. Die quartären Ablagerungen der Beckenfüllung nehmen die ganze Weite des 'Valley' ein. Lombard gibt einige Detailprofile, welche jedoch meines Erachtens den morpholo- 
gisch und geographisch relevanten Sachverhalt nicht genügend wiedergeben. Auch eine indische Arbeit ${ }^{6}$ trägt in dieser Hinsicht nicht viel zum Verständnis bei.

Südlich von Jawalakhel fließt der Nakhu Khola, ein linksseitiger Nebenfluß des Bagmati in breitem Tale dahin (Fig. 1; Beispiel 1). Figur 3 zeigt die Verhältnisse an der angegebenen Stelle in einem kleinen Seitentälchen. Der Nakhu Khola hat sich hier in die quartären Ablagerungen fast 100 m eingetieft. Die so aufgeschlossene Serie besteht zuoberst aus Konglomeraten, welche nach Größe und Verfestigungsgrad etwa mit unserem Hochterrassenschotter verglichen werden können (Vordergrund der Abbildung). Die Oberfläche ist eben und dürfte vermutlich mit der Akkumulationsoberfläche identisch sein; einen Beweis dafür fand ich aber nicht. Die Schotter sind 20 bis $30 \mathrm{~m}$ mächtig und stellen in ihrer Gesamtheit ein gewaltiges Grundwasserreservoir dar. Sie liegen einer grauen Mergelserie auf, welche als Wasserstauer dient und das Grundwasser an einer im obern Talhange verlaufenden Linie zum Austreten zwingt. Diese Linie ist in Fig. 3 markiert, ebenso mit einem Kreis die Lage eines Brunnens, an dem die Frauen Trinkwasser holen und waschen. Die Oberfläche des Schotterkomplexes ist während der Trokkenzeit wüstenhaft trocken und steinig, während der Regenzeit dient sie dem Regenfeldbau. Die Talhänge, soweit sie über dem Quellhorizont liegen, werden - falls nicht zu steil - als Weide genutzt und sind von horizontalen Viehweglein durchzogen. Unterhalb des Quellhorizontes vermag das ständig ausfließende Wasser den Boden im Grund des Seitentälchens zu durchfeuchten, und selbst in der Trockenzeit bleibt er begrünt. Während die Erde auf der Schotterfläche rötlich und an den trockenen, oberen Hängen gräulich verwittert, ist sie hier dunkelbraun und humusreich. Außerhalb des Talgrundes, im unteren Teil der sich im Bereich der Mergelschichten verflachenden Talhänge, werden Terrassen für Regenfeldbau angelegt. Die eigentlichen Bewässerungsterrassen, welchen das Wasser durch ein Kanalsystem zugeleitet wird, finden sich erst wenig über dem Haupttalboden; das Wasser wird dem Nakhu Khola entnommen.

Dieser Quellhorizont muß eine weite Verbreitung haben. Auf der Seite von Jawalakhel findet sich der Quellhorizont auf der Höhe des Tibeterlagers; Enten tummeln sich dort im Tümpel. In der alten Königstadt Patan gibt es schräg in die Tiefe führende alte Stollen, die den Quellhorizont für die Trinkwasserversorgung erschließen. Zweifellos könnte auch heute die Trinkwasserversorgung durch eine sorgfältige hydrogeologische Untersuchung wesentlich verbessert werden.

\section{MORPHOLOGICAL OBSERVATIONS IN EASTERN NEPAL}

In the article on «Morphological Observations in Eastern Nepal $v$ the author describes observations made along the main route from Kathmandu to Those in 1964 . This route is the main approach to the Mount Everest area. Figure 1 serves as location map, indicating with numbers $1-4$ the localities described in the text, namely: (1) a characteristic profile in the Kathmandu Valley, (2) the transversal profile typical for most of the valleys belonging to the Sun Kosi drainage system, (3a and $3 \mathrm{~b}$ ) old valley fillings of alluvial nature, (4) the special case of the liri Valley, which is not yet reached by retrogressive erosion. 